\title{
Cascade decays of heavy Higgs bosons through vectorlike quarks in two Higgs doublet models
}

\author{
Radovan Dermisek, ${ }^{a}$ Enrico Lunghi ${ }^{a}$ and Seodong Shin ${ }^{b, c, d, e}$ \\ ${ }^{a}$ Physics Department, Indiana University, \\ Bloomington, IN 47405, U.S.A. \\ ${ }^{b}$ Enrico Fermi Institute, University of Chicago, \\ Chicago, IL 60637, U.S.A. \\ ${ }^{c}$ Department of Physics and IPAP, Yonsei University, \\ Seoul 03722, Korea \\ ${ }^{d}$ Center for Theoretical Physics of the Universe, Institute for Basic Science, \\ Daejeon 34126, Korea \\ ${ }^{e}$ Department of Physics, Jeonbuk National University, \\ Jeonju, Jeonbuk 54896, Korea \\ E-mail: dermisek@indiana.edu, elunghi@indiana.edu, sshin@jbnu.ac.kr
}

ABSTRACT: We study cascade decays of heavy neutral Higgs bosons through vectorlike quarks. We focus on scenarios where decay modes into pairs of vectorlike quarks are not kinematically open which extends the sensitivity of the LHC to larger masses. Assuming only mixing with the third family of standard model quarks the new decay modes of heavy Higgs bosons are: $H \rightarrow t_{4} t \rightarrow W b t, Z t t, h t t$ and $H \rightarrow b_{4} b \rightarrow W t b, Z b b, h b b$, where $t_{4}\left(b_{4}\right)$ is the new up-type (down-type) quark mass eigenstate. In the numerical analysis we assume the CP even Higgs boson in the two Higgs doublet model type-II but the signatures are relevant for many other scenarios. We identify the region of the parameter space where these decay modes are significant or can even dominate, and thus they provide the best opportunities for the simultaneous discovery of a new Higgs boson and vectorlike quarks. We further explore the reach of the High Luminosity LHC for two representative decay modes, $t_{4} \rightarrow Z t \rightarrow \ell \ell t$ and $b_{4} \rightarrow Z b \rightarrow \ell \ell b$, and found that cross sections at a $0.1 \mathrm{fb}$ level can be probed with simple cut based analyses. We also find that the rates for Higgs cascade decays can be much larger than the rates for a single production of vectorlike quarks. Furthermore, the reach for vectorlike quarks in Higgs cascade decays and pair production extends to comparable masses.

Keywords: Beyond Standard Model, Heavy Quark Physics, Higgs Physics

ArXiv EPRINT: 1907.07188 


\section{Contents}

1 Introduction 1

2 Model 3

3 Parameter space scan and experimental constraints 4

4 Higgs production cross section and decays 5

5 Search strategies and comparison with single productions of $t_{4}$ and $b_{4} \quad 10$

6 Conclusions 13

$\begin{array}{ll}\text { A Partial decay widths of the heavy Higgs boson } & 15\end{array}$

\section{Introduction}

Among the simplest extensions of the standard model (SM) are models with extra Higgs bosons or vectorlike matter. Many searches for such individual new particles have been performed. However, if both sectors are present, new search strategies can be designed that could lead to a simultaneous discovery of heavy Higgs bosons and matter particles. These can be even more potent than separate searches. In this paper we focus on cascade decays of a heavy neutral Higgs boson through vectorlike quarks.

In this work, we consider an extension of the two Higgs doublet model type-II by vectorlike pairs of new quarks (VLQ), corresponding to a copy of the SM SU(2) doublet and singlet quarks and their vectorlike partners, introduced in ref. [1]. Assuming only the mixing with the third family of standard model quarks, the flavor changing couplings of $W, Z$ and Higgs bosons between new quarks and the third family quarks are generated. These couplings allow new decay modes of the heavy CP even (or CP odd) Higgs boson: $H \rightarrow t_{4} t$ and $H \rightarrow b_{4} b$, where $t_{4}$ and $b_{4}$ are the lightest new up-type and down-type quark mass eigenstates. Although these decay modes compete with $H \rightarrow t \bar{t}$ and $H \rightarrow b \bar{b}$ we will see that, in a region of the parameter space, they are significant or can even dominate. Here we assume that the light Higgs boson $(h)$ is SM-like so that $H \rightarrow Z Z, W W$ are not present. Subsequent decay modes of $t_{4}$ and $b_{4}: t_{4} \rightarrow W b, t_{4} \rightarrow Z t, t_{4} \rightarrow h t$ and $b_{4} \rightarrow W t$, $b_{4} \rightarrow Z b, b_{4} \rightarrow h b$ lead to the following 6 decay chains of the heavy Higgs boson:

$$
\begin{aligned}
& H \rightarrow t_{4} t \rightarrow W b t, Z t t, h t t, \\
& H \rightarrow b_{4} b \rightarrow W t b, Z b b, h b b,
\end{aligned}
$$

which are also depicted in figure 1. 


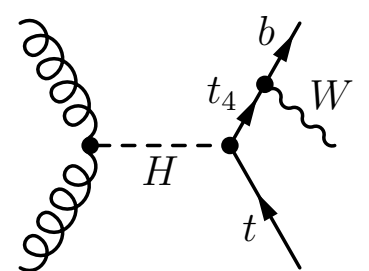

(a)

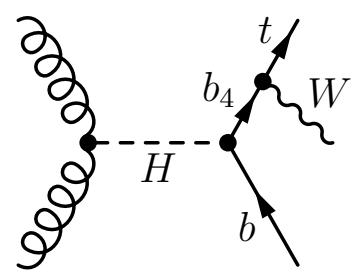

(d)

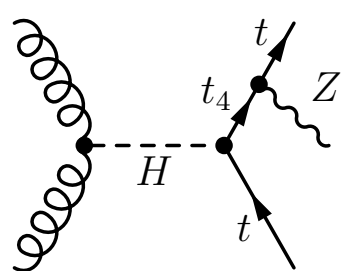

(b)

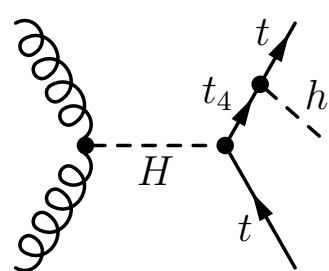

(c)

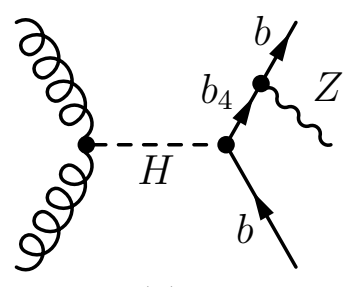

(e)

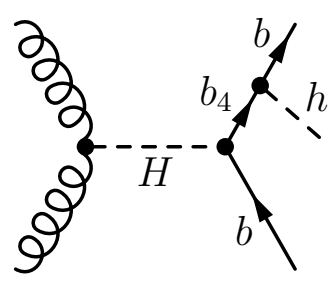

(f)

Figure 1. New decay topologies of a heavy Higgs boson through vectorlike quarks.

In addition, a heavy neutral Higgs boson could also decay into pairs of vectorlike quarks. However, we focus on the range of masses where $H \rightarrow t_{4} \bar{t}_{4}, b_{4} \bar{b}_{4}$ are not kinematically open which extends the sensitivity of the LHC to larger masses of vectorlike quarks (moreover, the final states would be the same as for the pair production of vectorlike quarks). We also find that the rates for processes (1.1) and (1.2) can be much larger than the rates for a single production of vectorlike quarks. Thus, in the identified regions, they provide the best opportunities for the discovery of a new Higgs boson and vectorlike quarks. Although in the numerical analysis we assume the heavy CP even Higgs boson in the two Higgs doublet model type-II, the signatures are relevant for many other scenarios.

Similar signatures in the lepton sector, cascade decays of heavy Higgs bosons through vectorlike leptons, were studied in refs. [2-6]. If decay modes through both vectorlike quarks and leptons are kinematically open, the decays through quarks are expected to dominate because of the color factor. On the other hand, decay modes through leptons provide several very clean signatures $[4,7]$ that might compensate for smaller rates. Alternatively, in the same model, if vectorlike quarks or leptons are heavier, they can decay through heavy Higgs bosons, including the charged Higgs boson, leading to very rare final states. The corresponding signatures were recently studied in ref. [1].

Vectorlike quarks and leptons near the electroweak scale provide a very rich phenomenology and often they are introduced to explain various anomalies. Examples include discrepancies in precision Z-pole observables [8-11] and the muon g-2 anomaly [12, 13] among many others. They are also considered for a variety of theoretical reasons. Examples include studies of their effects on gauge and Yukawa couplings in the framework of grand unification [14-22], on electroweak symmetry breaking [23], and the Higgs boson mass and its decays [24-31]. The supersymmetric extension with a complete vectorlike family also provides a possibility to understand the values of all large couplings in the SM from the IR fixed point structure of the renormalization group equations [32]. The possibility of 


\begin{tabular}{|ccccccccc|}
\hline & $q_{L}$ & $t_{R}$ & $d_{R}$ & $Q_{L, R}$ & $T_{L, R}$ & $B_{L, R}$ & $H_{d}$ & $H_{u}$ \\
\hline $\mathrm{SU}(2)_{\mathrm{L}}$ & $\mathbf{2}$ & $\mathbf{1}$ & $\mathbf{1}$ & $\mathbf{2}$ & $\mathbf{1}$ & $\mathbf{1}$ & $\mathbf{2}$ & $\mathbf{2}$ \\
$\mathrm{U}(1)_{\mathrm{Y}}$ & $\frac{1}{6}$ & $\frac{2}{3}$ & $-\frac{1}{3}$ & $\frac{1}{6}$ & $\frac{2}{3}$ & $-\frac{1}{3}$ & $\frac{1}{2}$ & $-\frac{1}{2}$ \\
$\mathrm{Z}_{2}$ & + & + & - & + & + & - & - & + \\
\hline
\end{tabular}

Table 1. Quantum numbers of the 3 rd generation standard model quarks $\left(q_{L}, t_{R}, d_{R}\right)$, extra vectorlike quarks and the two Higgs doublets. The electric charge is given by $Q=T_{3}+Y$, where $T_{3}$ is the weak isospin, which is $+1 / 2$ for the first component of a doublet and $-1 / 2$ for the second component.

a simple embedding into grand unified theories is one of the main motivations for considering complete copies of SM families. We should note, however, that vectorlike quarks with other quantum numbers have been considered in the literature (see, for instance, ref. [33]). Vectorlike fermions have also been studied in composite Higgs models [34-47], little Higgs models [48, 49] and models with a gauged flavor group [50-53]. Most of these scenarios focus on mixing between the new vectorlike fermions and the third generation of SM quarks and lead to some generic signatures (see, for instance, refs. [33, 54-57]).

This paper is organized as follows. In section 2 we briefly summarize the model. Details of the analysis and experimental constraints are discussed in section 3. The main results are presented in section 4 . We briefly discuss the search strategies in section 5 and conclude in section 6 . The appendix contains formulas for partial decay widths of the heavy Higgs boson.

\section{Model}

We consider an extension of the two Higgs doublet model type-II by vectorlike pairs of new quarks: $\mathrm{SU}(2)$ doublets $Q_{L, R}$ and $\mathrm{SU}(2)$ singlets $T_{L, R}$ and $B_{L, R}$. The $Q_{L}, T_{R}$ and $B_{R}$ have the same quantum numbers as the SM quark doublet $q_{L}$ and the right-handed quark singlets $u_{R}$ and $d_{R}$, respectively. The quantum numbers of new quarks, SM quarks and two Higgs doublets, are summarized in table 1. The model is described in detail in ref. [1] and thus we just briefly summarize it here.

As is characteristic for the two Higgs doublet model type-II, we assume that the down sector couples to $H_{d}$ and the up sector couples to $H_{u}$. This can be achieved by the $Z_{2}$ symmetry specified in table 1 . The generalization to the whole vectorlike family of new fermions, including the lepton sector introduced in ref. [2], is straightforward. We further assume that, in the basis in which the SM quark Yukawa couplings are diagonal, the new quarks mix only with one family of SM quarks and we consider the mixing with the third family as an example. An arbitrary mixing could be easily accommodated.

The most general renormalizable Lagrangian consistent with our assumptions contains the following Yukawa and mass terms for the SM and vectorlike quarks:

$$
\begin{aligned}
\mathcal{L} \supset & -y_{b} \bar{q}_{L} d_{R} H_{d}-\lambda_{B} \bar{q}_{L} B_{R} H_{d}-\lambda_{Q} \bar{Q}_{L} d_{R} H_{d}-\lambda \bar{Q}_{L} B_{R} H_{d}-\bar{\lambda} H_{d}^{\dagger} \bar{B}_{L} Q_{R} \\
& -y_{t} \bar{q}_{L} t_{R} H_{u}-\kappa_{T} \bar{q}_{L} T_{R} H_{u}-\kappa_{Q} \bar{Q}_{L} u_{R} H_{u}-\kappa \bar{Q}_{L} T_{R} H_{u}-\bar{\kappa} H_{u}^{\dagger} \bar{T}_{L} Q_{R} \\
& -M_{Q} \bar{Q}_{L} Q_{R}-M_{T} \bar{T}_{L} T_{R}-M_{B} \bar{B}_{L} B_{R}+\text { h.c. },
\end{aligned}
$$


where the first term is the bottom Yukawa coupling, followed by Yukawa couplings of vectorlike quarks to $H_{d}$ (denoted by various $\lambda \mathrm{s}$ ), the top Yukawa coupling, Yukawa couplings of vectorlike quarks to $H_{u}$ (denoted by various $\kappa \mathrm{s}$ ), and finally by mass terms for vectorlike quarks. Note that the explicit mass terms mixing SM and vectorlike quarks, $M_{q} \bar{q}_{L} Q_{R}$, $M_{t} \bar{T}_{L} t_{R}$ and $M_{b} \bar{B}_{L} b_{R}$, can be removed by redefinitions of $Q_{L}, T_{R}, B_{R}$ and the Yukawa couplings. The components of doublets are labeled as follows:

$$
q_{L}=\left(\begin{array}{c}
t_{L} \\
b_{L}
\end{array}\right), Q_{L, R}=\left(\begin{array}{c}
T_{L, R}^{Q} \\
B_{L, R}^{Q}
\end{array}\right), H_{d}=\left(\begin{array}{c}
H_{d}^{+} \\
H_{d}^{0}
\end{array}\right), H_{u}=\left(\begin{array}{c}
H_{u}^{0} \\
H_{u}^{-}
\end{array}\right) .
$$

We assume that the neutral Higgs components develop real and positive vacuum expectation values, $\left\langle H_{u}^{0}\right\rangle=v_{u}$ and $\left\langle H_{d}^{0}\right\rangle=v_{d}$, as in the $C P$ conserving two Higgs doublet model with $\sqrt{v_{u}^{2}+v_{d}^{2}}=v=174 \mathrm{GeV}$ and we define $\tan \beta \equiv v_{u} / v_{d}$. Plugging the vacuum expectation values to the Lagrangian, we obtain the mass matrices describing the mixing between the third generation and vectorlike quarks:

$$
\begin{aligned}
\left(\begin{array}{lll}
\bar{t}_{L} & \bar{T}_{L}^{Q} & \bar{T}_{L}
\end{array}\right) M_{t}\left(\begin{array}{c}
t_{R} \\
T_{R}^{Q} \\
T_{R}
\end{array}\right) & =\left(\begin{array}{lll}
\bar{t}_{L} & \bar{T}_{L}^{Q} & \bar{T}_{L}
\end{array}\right)\left(\begin{array}{ccc}
y_{t} v_{u} & 0 & \kappa_{T} v_{u} \\
\kappa_{Q} v_{u} & M_{Q} & \kappa v_{u} \\
0 & \bar{\kappa} v_{u} & M_{T}
\end{array}\right)\left(\begin{array}{c}
t_{R} \\
T_{R}^{Q} \\
T_{R}
\end{array}\right), \\
\left(\begin{array}{ccc}
\bar{b}_{L} & \bar{B}_{L}^{Q} & \bar{B}_{L}
\end{array}\right) M_{b}\left(\begin{array}{c}
b_{R} \\
B_{R}^{Q} \\
B_{R}
\end{array}\right) & =\left(\begin{array}{lll}
\bar{b}_{L} & \bar{B}_{L}^{Q} & \bar{B}_{L}
\end{array}\right)\left(\begin{array}{ccc}
y_{b} v_{d} & 0 & \lambda_{B} v_{d} \\
\lambda_{Q} v_{d} & M_{Q} & \lambda v_{d} \\
0 & \bar{\lambda} v_{d} & M_{B}
\end{array}\right)\left(\begin{array}{c}
b_{R} \\
B_{R}^{Q} \\
B_{R}
\end{array}\right) .
\end{aligned}
$$

We label the resulting mass eigenstates as $t_{i}$ and $b_{i}$ with $i=3,4,5$, where $t_{3}$ and $b_{3}$ represent the top quark and the bottom quark. A complete discussion of mass eigenstates, their couplings to the $W, Z$, and Higgs bosons, various approximate formulas, and other details can be found in ref. [1]. Formulas for partial decay widths of the heavy Higgs boson are summarized in the appendix. In the following section, we perform the numerical analysis assuming the alignment limit in which the light Higgs coupling to gauge bosons are identical to those in the SM. This is most easily, but not necessarily, attained in the decoupling limit where all the non-SM Higgs bosons are heavy [58-60].

\section{Parameter space scan and experimental constraints}

In the numerical study we scan the parameters of the model in the following ranges:

$$
\begin{aligned}
M_{Q, T, B} & \in[900,4000] \mathrm{GeV}, \\
\kappa_{T}, \kappa_{Q}, \kappa, \bar{\kappa} & \in[-1.0,1.0], \\
\lambda_{B}, \lambda_{Q}, \lambda, \bar{\lambda} & \in[-1.0,1.0], \\
\tan \beta & \in[0.3,50],
\end{aligned}
$$

and we will also comment on the impact of lowering the upper ranges in eqs. (3.2) and (3.3). Note that the signs of three Yukawa couplings are not physical and can be absorbed into 
a redefinition of the three vectorlike quark fields (any set of three couplings not containing both $\lambda_{Q}$ and $\kappa_{Q}$ can be chosen to be positive). For each choice of the parameters in eqs. (3.1)-(3.4), $y_{t}$ and $y_{b}$ are determined iteratively to reproduce the top and bottom masses.

Due to current constraints on vectorlike quark masses, all indirect constraints have a minimal impact on our scan. We impose experimental constraints from oblique corrections and $R_{b}$ [61] following the results presented in section II of ref. [62] and section IIIB of ref. [63] (see also refs. [41, 64]). We further include bounds from $h \rightarrow(\gamma \gamma, 4 \ell)$ [65-67] (note that in the calculation of the effective $h g g$ coupling we include effects of vectorlike quarks, that can be found, for instance, in refs. [68, 69]), and direct searches for pair production of vectorlike quarks at the LHC [70, 71] (for each scan point we calculate the $t_{4}$ and $b_{4}$ branching ratios into $W, Z$ and $h$ and compare with the constraint). We do not use searches for single production of VLQ $[72,73]$ since the constraints are not stronger than those from the pair production. We finally impose searches for heavy Higgs bosons: $H \rightarrow \tau^{+} \tau^{-}[74,75], H \rightarrow \gamma \gamma[76,77]$. In the alignment limit, the width $H \rightarrow h h$ is given in eq. (A.7) which impacts the $H \rightarrow h h$ branching ratio at the $O(0.1 \%)$ level; hence $H \rightarrow h h$ constraints [78] are negligible.

\section{Higgs production cross section and decays}

Let us start by discussing the heavy neutral CP-even Higgs boson production cross section. Note that the results for the case of a CP-odd Higgs $(A)$ would be very similar. In fact, the $b b H$ and $b b A$ production cross sections, which dominate at large $\tan \beta$, are essentially identical, and the gluon fusion production of $A$ is only slightly larger than the $H$ one for large Higgs masses (see, for instance, figure 3.36 of ref. [68]). Also, in the alignment limit, the $H$ and $A$ branching ratios are almost identical.

In figure 2, we show the production cross section dependence on the Higgs mass and $\tan \beta$ for scenarios in which the $H \rightarrow\left(t_{4} t, b_{4} b\right)$ decays are kinematically open and satisfy all experimental constraints. The lower bound on the Higgs mass in the left panel of figure 2 is thus connected to the limits from direct searches for vectorlike quarks. This scenario is also constrained by $H \rightarrow \tau^{+} \tau^{-}$searches that, for large $\tan \beta$, extend to larger Higgs masses.

The effective $g g H$ vertex is dominated by top and bottom loops, which give contributions almost identical to the type-II two Higgs doublet model ones. Vectorlike quark loops generate the spread at large Higgs mass and $\operatorname{small} \tan \beta$. The lower bounds on vectorlike quark masses imply that the $g g H$ vertex is significantly affected only for very heavy Higgs masses (in general one expects the largest effects for $m_{H} \sim 2 m_{t_{4}, b_{4}}$, see, for instance, ref. [69]). To understand the $\tan \beta$ dependence, we note that $\lambda_{t_{4} t_{4}}^{H} \propto v_{u} \cos \beta \propto \sin 2 \beta$ and that $\lambda_{b_{4} b_{4}}^{H} \propto v_{d} \sin \beta \propto \sin 2 \beta$, implying that both are the largest at $\tan \beta \sim 1$. The actual cross sections are calculated using SusHi [79] and then rescaled to take into account the impact of the modified $g g H$ vertex.

In figure 3 we show the $\tan \beta$ dependence of Higgs partial decay widths and branching ratios for scenarios with couplings to $H_{u}$ only (all $\lambda \mathrm{s}=0$ ), $H_{d}$ only (all $\kappa_{\mathrm{s}}=0$ ) and to both $H_{u}$ and $H_{d}$. The dominant features of these plots can be easily understood from the 

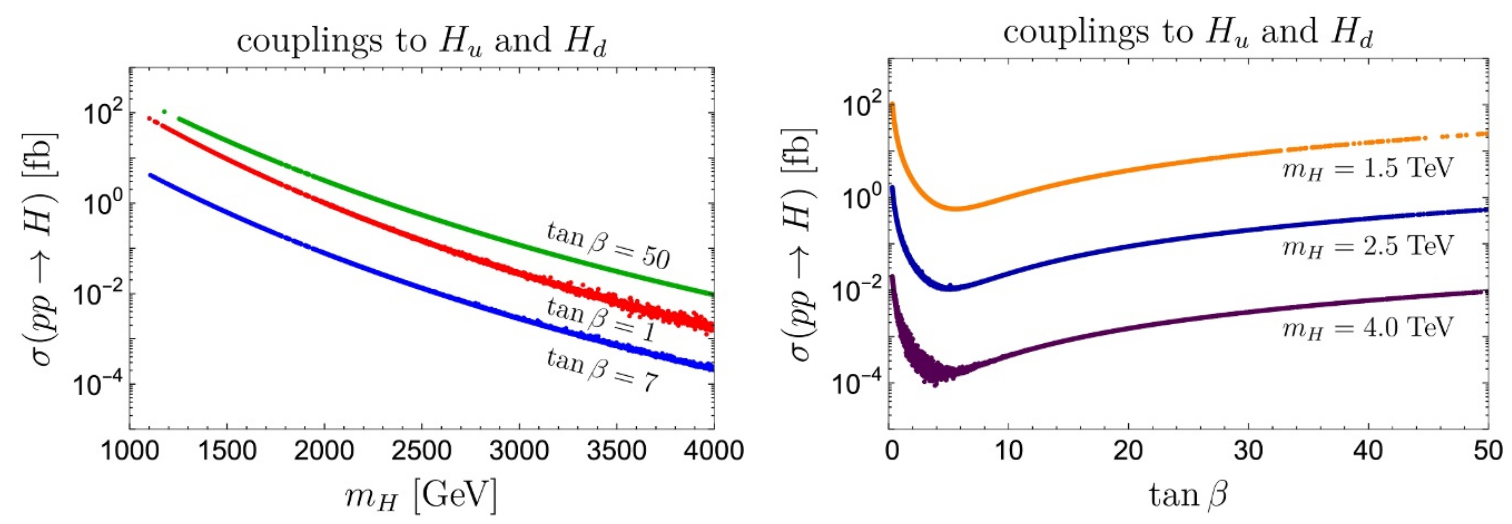

Figure 2. Production cross section of a heavy neutral Higgs boson in scenarios in which decays through a vectorlike quark are open as a function of $m_{H}$ for $\tan \beta=1,7$ and 50 (left) and as a function of $\tan \beta$ for $m_{H}=1.5,2.5$ and $4 \mathrm{TeV}$ (right).

$\tan \beta$ dependence of the heavy Higgs couplings to $t$ and $t_{4}: \lambda_{t t}^{H} \propto 1 / \tan \beta$ and $\lambda_{t_{4} t}^{H} \propto \cos \beta$, and couplings to $b$ and $b_{4}: \lambda_{b b}^{H} \propto \tan \beta$ and $\lambda_{b_{4} b}^{H} \propto \sin \beta$ (see table 2 of ref. [1]). These $\tan \beta$ dependences directly translate into the dependence of the partial widths in the left panels of figure 3 .

In the scenario with couplings to $H_{u}$ only, the $H \rightarrow t_{4} t$ mode is asymptotically smaller than both $H \rightarrow t \bar{t}$ (at small $\tan \beta$ ) and $H \rightarrow b \bar{b}$ (at large $\tan \beta$ ) and is relevant only at small-to-medium $\tan \beta$ : we find branching ratios larger than $10 \%$ for $\tan \beta \in[0.5,10]$ and they can reach up to $40 \%$. On the other hand, in the scenario with couplings to $H_{d}$, the $H \rightarrow b_{4} b$ mode is still asymptotically smaller than $H \rightarrow t \bar{t}$ at small $\tan \beta$ but is not suppressed with respect to $H \rightarrow b \bar{b}$ at large $\tan \beta$. We find branching ratios larger than $10 \%$ for any $\tan \beta>0.8$. More importantly, this mode can dominate for $\tan \beta \in[4,18]$ and can reach up to $95 \%$. The scenario with couplings to both $H_{u}$ and $H_{d}$ can be understood in a similar way. ${ }^{1}$

Note that the maximum Higgs partial widths and branching ratios into vectorlike quarks depend on the ranges of Yukawa couplings that we scan over that are given in eqs. (3.2) and (3.3). The maximum partial widths scale with the square of the maximum coupling allowed; for example, limiting the upper ranges to 0.5 reduces the maximum widths by a factor of 4 . The impact on the branching ratios is less straightforward. Reducing the upper range of the scan to 0.5 implies that the $H \rightarrow t_{4} t$ branching ratio peaks at $15 \%$; the $H \rightarrow b_{4} b$ branching ratio dominates for $\tan \beta \in[5,10]$, peaks at about $85 \%$ but drops to about $20 \%$ for $\tan \beta \sim 50$.

Due to different $\tan \beta$ dependence of Higgs production cross section and branching ratios, it is interesting to show the total rate into individual final states. In figure 4 we show the various production rates for $m_{H}=2.5 \mathrm{TeV}$ as functions of $\tan \beta$. We see that the $t_{4} t$ mode is the largest at very small $\tan \beta$ and that, although the $H \rightarrow b_{4} b$ mode can dominate at medium $\tan \beta$, the $\sigma\left(p p \rightarrow H \rightarrow b_{4} b\right)$ can still be larger at both small and

\footnotetext{
${ }^{1}$ In the calculation of the branching ratios we account for decays through both $t_{4}\left(b_{4}\right)$ and $t_{5}\left(b_{5}\right)$ when kinematically open. This effectively reduces the branching ratio into $t_{4}\left(b_{4}\right)$.
} 

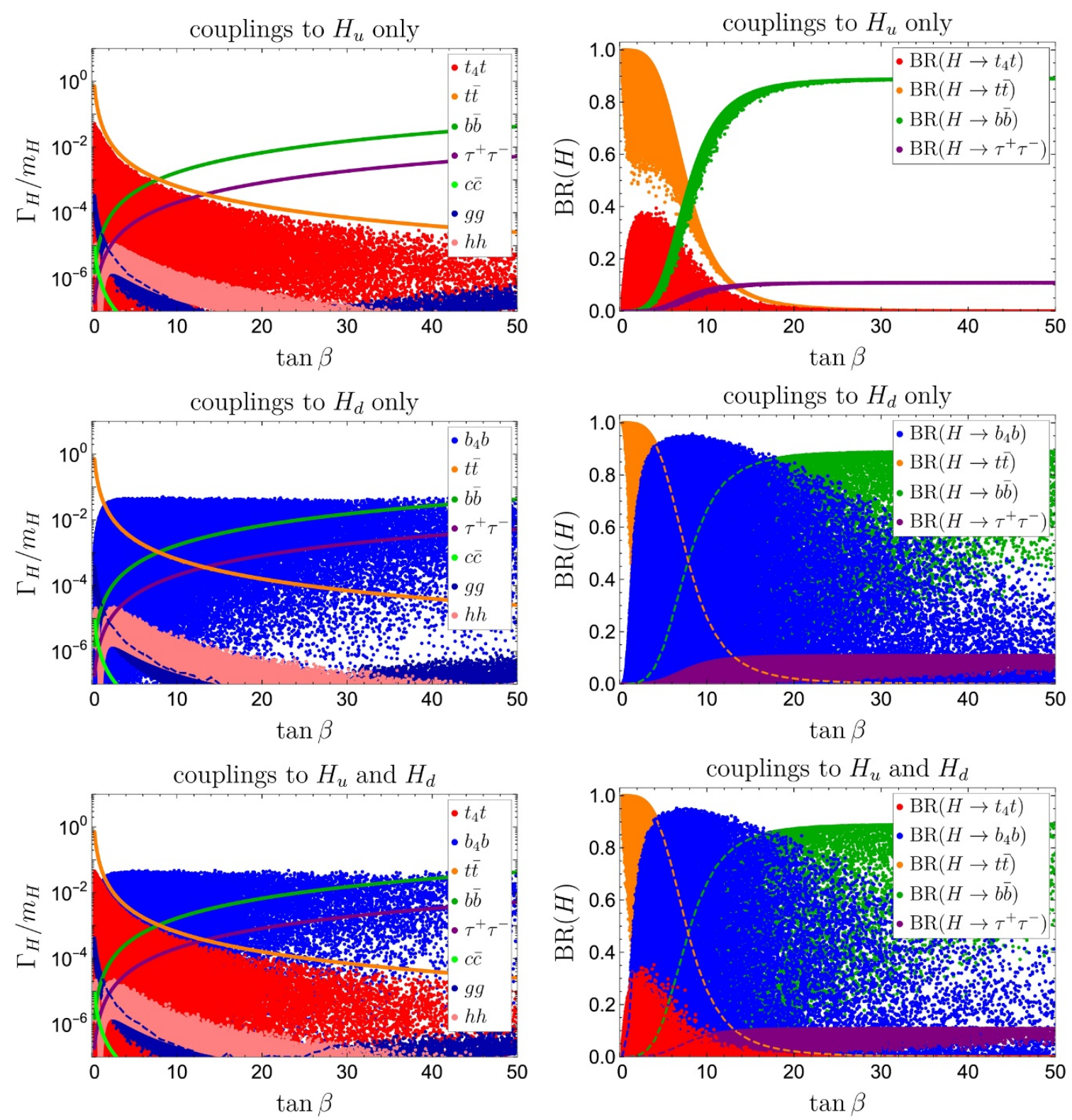

Figure 3. Partial widths (left) and branching ratios (right) of a heavy neutral Higgs boson as functions of $\tan \beta$ assuming couplings to $H_{u}$ only (top), $H_{d}$ only (middle), and $H_{u}$ and $H_{d}$ (bottom).

very large $\tan \beta$. Rates of the order of $0.1 \mathrm{fb}$ are attainable for $H \rightarrow t_{4} t$ at very small $\tan \beta$ and for $H \rightarrow b_{4} b$ at medium-to-large $\tan \beta$.

The lightest new quarks from heavy Higgs decays further decay into SM particles. The correlations between the branching ratio of $H \rightarrow t_{4} t$ and individual branching ratios of $t_{4}$ are shown in figure 5 and similar correlations for $b_{4}$ are shown in figure 6 . Main features of these plots can be understood from approximate formulas and the discussion in ref. [1]. We see that the decay modes of $t_{4}$ and $b_{4}$ into $W, Z$ and $h$, cluster around the pattern expected from the Goldstone boson equivalence limit corresponding to sending all vectorlike quark 


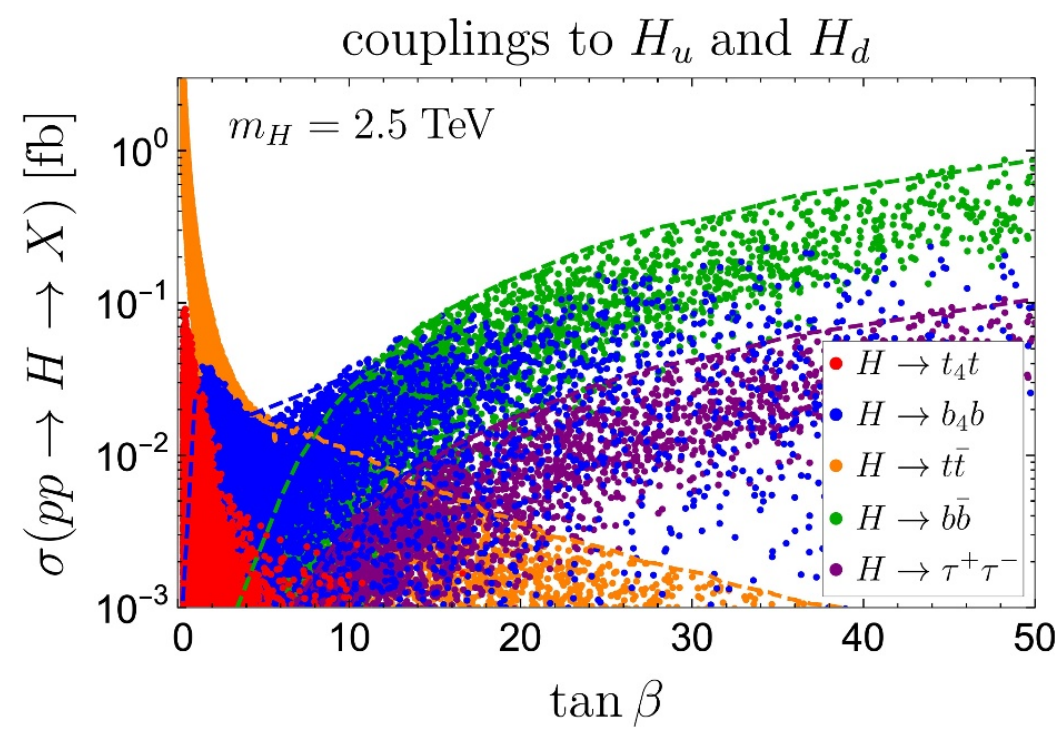

Figure 4. Production cross section of a heavy neutral Higgs boson multiplied by branching ratios to individual decay modes as functions of $\tan \beta$ for $m_{H}=2.5 \mathrm{TeV}$.

masses to infinity. For singlet-like new quarks (red) ${ }^{2}$ this leads to 2:1:1 branching ratios into $W, Z$ and $h$. For doublet-like new quarks (blue) this leads to a one parameter family of branching ratios characterized by an arbitrary branching ratio to $W$ and equal branching ratios to $Z$ and $h$. For example, for a doublet-like $t_{4}$, the $W t_{4} t$ coupling is controlled by $\lambda_{Q}$ while the corresponding couplings to $Z$ and both Higgs bosons are controlled by $\kappa_{Q}$. This results in a difference between the plots on the top (no couplings to $H_{d}$ allowed) and bottom (all couplings allowed) in figure 5 and similarly for the $b_{4}$ in figure 6 . The main distinction between the corresponding plots in figures 5 and 6 originates from different $\tan \beta$ dependence of relevant couplings. Note especially that while the branching ratios for $t_{4} \rightarrow W b$ and $b_{4} \rightarrow W t$ extend to $100 \%$ in the scenario with couplings to both $H_{u}$ and $H_{d}$, the former anticorrelates with the $H \rightarrow t_{4} t$ branching ratio as can be seen in the lower-left panel of figure 5 .

The mixed scenarios (cyan and purple) interpolate between mostly singlet and mostly doublet cases. Note that these scenarios require careful choices of model parameters especially for $b_{4}$ at medium to large $\tan \beta$, see eq. (2.4), where $H \rightarrow b_{4} b$ is sizable. This is the reason for an empty area in between the mostly singlet and mostly doublet cases in the top plots of figure 6 . It is expected that with large statistics the whole area would be populated.

Finally, as discussed in detail in ref. [1], with the general structure of Yukawa matrices that we allow, essentially arbitrary branching ratios of $t_{4}$ and $b_{4}$ can be achieved. However, going away from the Goldstone boson equivalence limit correlates with diminishing $H \rightarrow t_{4} t$ and $H \rightarrow b_{4} b$ because it requires very small $\kappa_{Q, T}$ and $\lambda_{Q, B}$ couplings that are directly related to $\mathrm{Ht}_{4} t$ and $\mathrm{Hb}_{4} b$ couplings.

\footnotetext{
${ }^{2}$ Singlet and doublet fractions of new quarks are defined in ref. [1].
} 

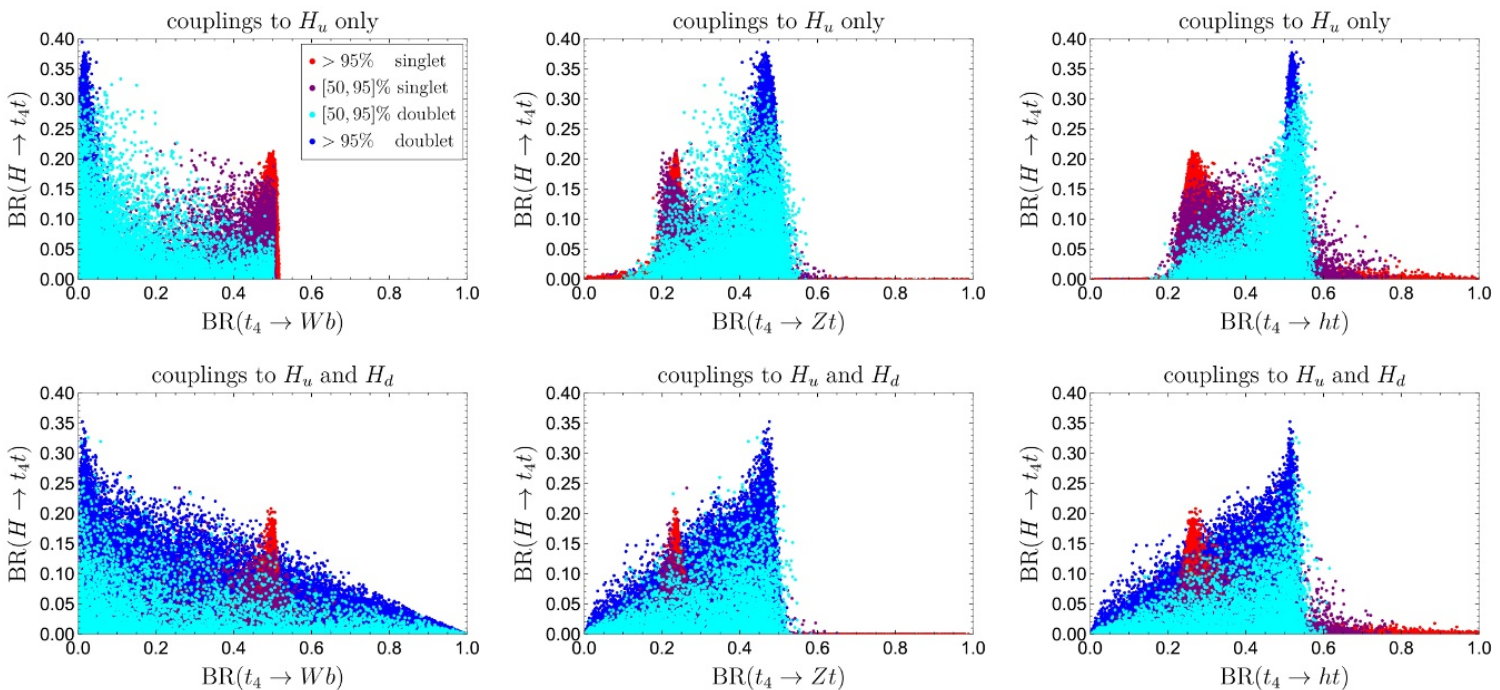

Figure 5. Correlations between the branching ratio of $H \rightarrow t_{4} t$ and branching ratios of $t_{4}$ assuming couplings to $H_{u}$ only (top) and couplings to $H_{u}$ and $H_{d}$ (bottom). Different colors correspond to different singlet/doublet fractions of $t_{4}$.
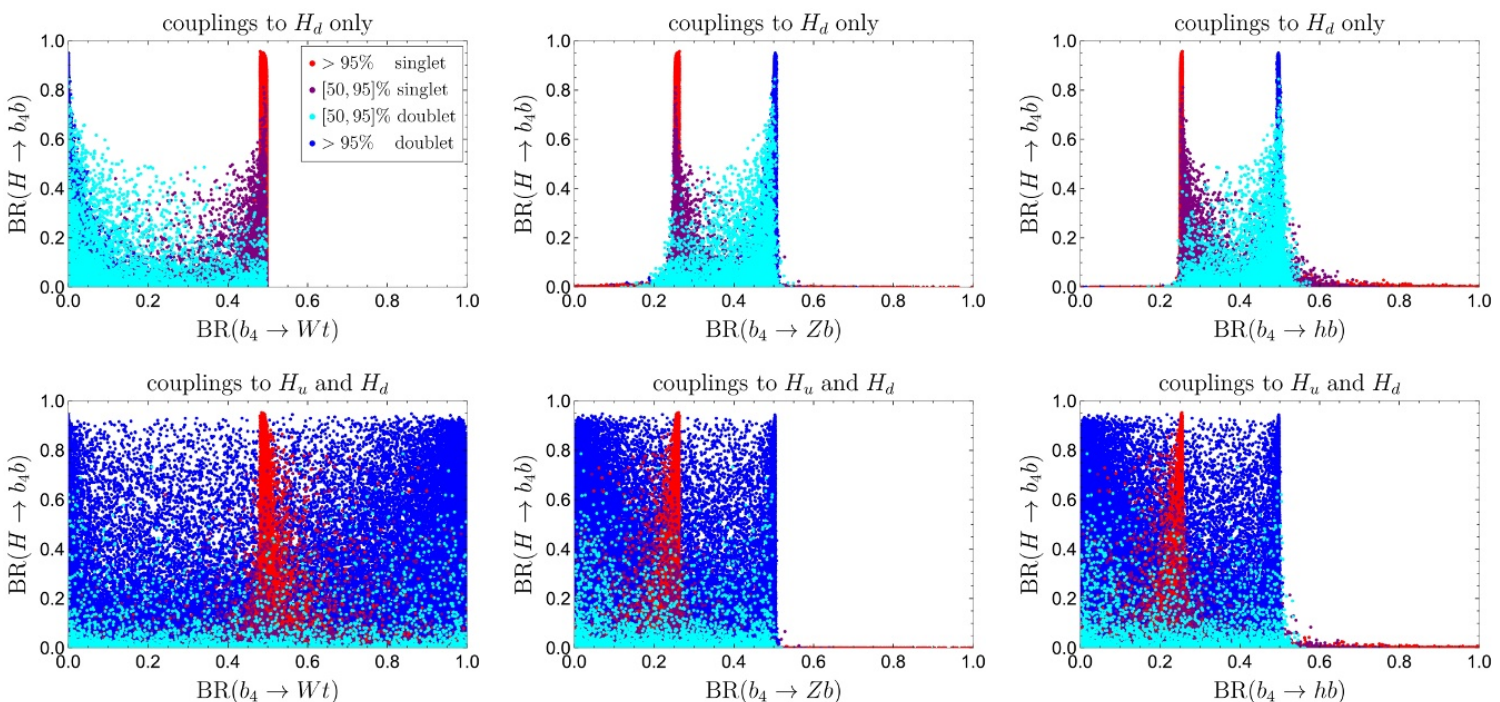

Figure 6. Correlations between the branching ratio of $H \rightarrow b_{4} b$ and branching ratios of $b_{4}$ assuming couplings to $H_{d}$ only (top) and couplings to $H_{u}$ and $H_{d}$ (bottom). Different colors correspond to different singlet/doublet fractions of $b_{4}$.

The maximum production rates of individual final states in cascade decays of a heavy Higgs boson as functions of $m_{H}$ and $m_{t_{4}}$ or $m_{b_{4}}$ are presented in figure 7 . We see that, for Higgs cascade decays through a $t_{4}$, rates of $0.1 \mathrm{fb}$ extend up to $m_{H} \lesssim 2 \mathrm{TeV}$ and $m_{t_{4}} \lesssim 1.4 \mathrm{TeV}$; rates above $1 \mathrm{ab}$ can be achieved for $m_{H} \lesssim 3.5 \mathrm{TeV}$ or $m_{t_{4}} \lesssim 2.5 \mathrm{TeV}$. For Higgs cascade decays through a $b_{4}$, rates of individual final states larger than $0.1 \mathrm{fb}$ extend up to $m_{H} \lesssim 2.5 \mathrm{TeV}$ and $m_{b_{4}} \lesssim 1.8 \mathrm{TeV}$ and can be even larger than $1 \mathrm{fb}$ for $m_{H} \lesssim 1.6 \mathrm{TeV}$ and $m_{b_{4}} \lesssim 1.2 \mathrm{TeV}$; rates above $1 \mathrm{ab}$ can be achieved for $m_{H} \lesssim 4 \mathrm{TeV}$ or $m_{b_{4}} \lesssim 3 \mathrm{TeV}$. 

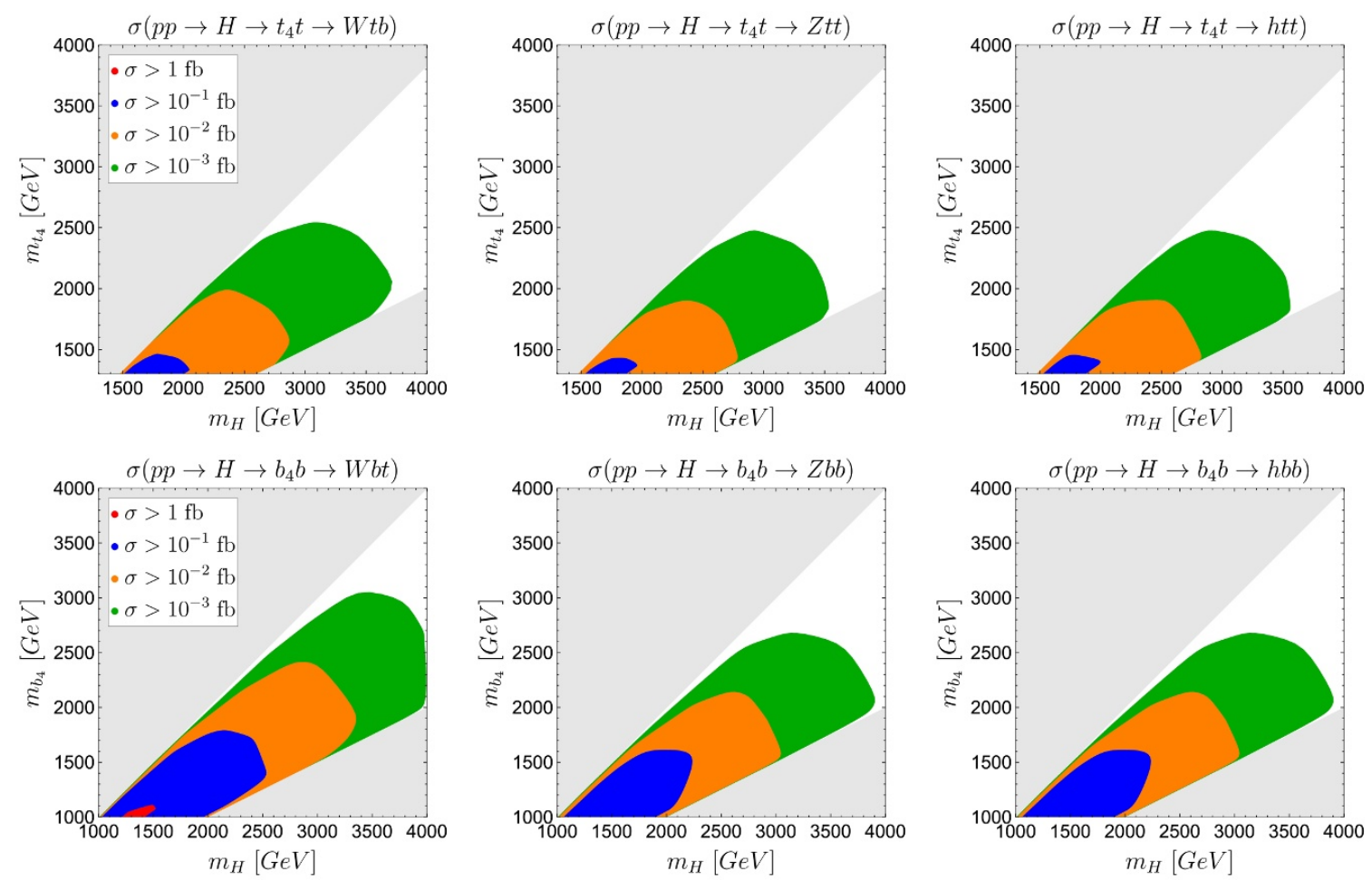

Figure 7. Maximum production rates of individual final states in cascade decays of a heavy Higgs boson as functions of $m_{H}$ and $m_{t_{4}}$ (top panels) or $m_{b_{4}}$ (bottom panels) in the scenario with couplings to both $H_{u}$ and $H_{d}$. In gray shaded regions $H \rightarrow t_{4} t, b_{4} b$ modes are not kinematically allowed or $H \rightarrow t_{4} \bar{t}_{4}, b_{4} \bar{b}_{4}$ modes are open.

\section{Search strategies and comparison with single productions of $t_{4}$ and $b_{4}$}

The signatures of cascade decays of a heavy Higgs boson through vectorlike quarks are almost identical to the production of any new resonance (e.g. $Z^{\prime}$ ) decaying to $t_{4} t$ or $b_{4} b{ }^{3}$ In addition, the final states we consider are very similar to the single production of both top-like and bottom-like vectorlike quarks, therefore all searches for a singly produced vectorlike quark can be reinterpreted as bounds on Higgs cascade decays. Note however that the topology of cascade decays provides more handles. For example, in the $t_{4}$ case, there is a top quark in the decay chain, and, in all cases, there is an additional resonance at the heavy Higgs mass. Thus, dedicated searches have a potential to considerably improve the limits found in standard single production studies.

Searches for $Z^{\prime} \rightarrow t_{4} t$ by CMS have been presented in refs. [83, 84] using $35.9 \mathrm{fb}^{-1}$ of integrated luminosity. In ref. [83] a search for $p p \rightarrow t_{4} b j \rightarrow Z t t$ with $Z \rightarrow \ell \ell$ and hadronic top, recasted as the production and decay of a $Z^{\prime}$, found bounds in the range $0.06-0.13 \mathrm{pb}$. In ref. [84] a dedicated search for $p p \rightarrow Z^{\prime} \rightarrow t_{4} t \rightarrow(W b, Z t, h t) t$ placed bounds in the range 10-100 fb in the lepton plus jets final state for $m_{t_{4}}<3 \mathrm{TeV}$ and $m_{Z^{\prime}}<4 \mathrm{TeV}$.

\footnotetext{
${ }^{3}$ Similar signatures also appeared in the context of composite Higgs models [80, 81] and extra dimensions [82].
} 

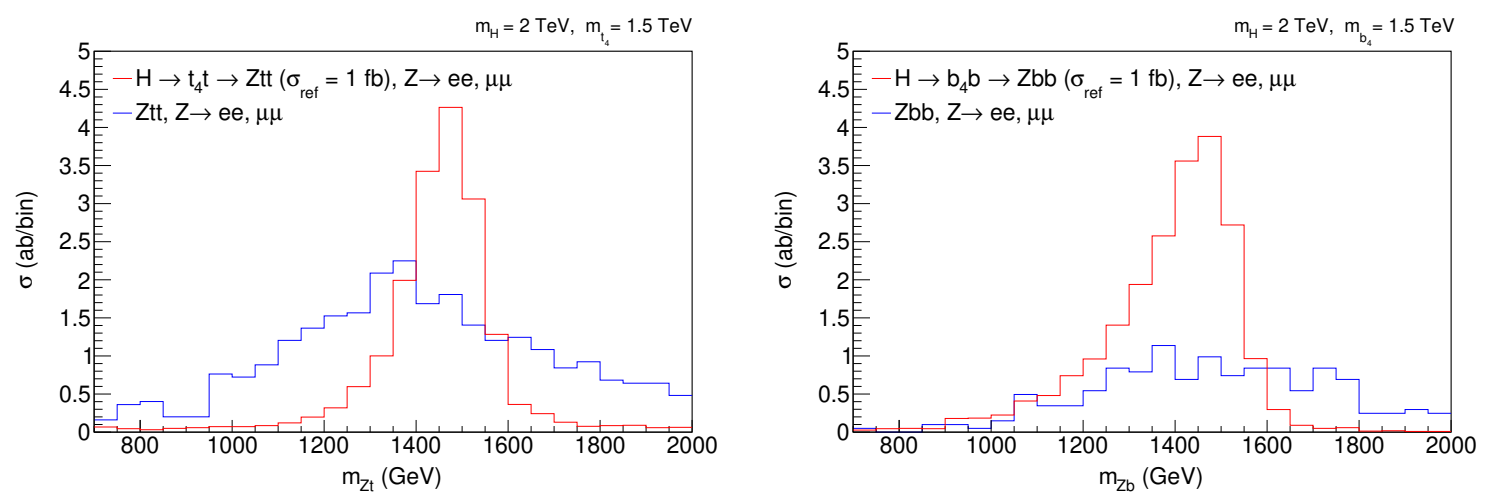

Figure 8. Left panel: distribution of the invariant mass of the di-lepton pair plus the hardest fatjet for the $H \rightarrow t_{4} t \rightarrow Z t t$ signal and the corresponding $Z t t$ background, with $Z \rightarrow \ell \ell(\ell=e, \mu)$. Right panel: distribution of the invariant mass of the di-lepton pair plus the hardest b-jet for the $H \rightarrow b_{4} b \rightarrow Z b b$ signal and the corresponding $Z b b$ background, with $Z \rightarrow \ell \ell(\ell=e, \mu)$. We take $m_{H}=2 \mathrm{TeV}, m_{t_{4}, b_{4}}=1.5 \mathrm{TeV}$ and $\sigma\left(p p \rightarrow H \rightarrow t_{4} t \rightarrow Z t t\right)=\sigma\left(p p \rightarrow H \rightarrow b_{4} b \rightarrow Z b b\right)=1 \mathrm{fb}$.

An important result of our analysis is that the rates for cascade decays through a bottom-like vectorlike quark $\left(b_{4}\right)$ can be almost an order of magnitude larger than the rates for cascade decays through the top-like vectorlike quark $\left(t_{4}\right)$. However, dedicated searches or recasted analyses for a resonance decaying to $b_{4} b$ have not been performed. Searches for the single production of bottom-like vectorlike quark in the $W t$ and $h b$ final states by CMS have been presented in refs. $[85,86]$ where bounds in the $0.1-1 \mathrm{pb}$ were found. ATLAS studies of singly produced top- and bottom-like vectorlike quarks have been presented in refs. $[73,87,88]$ where bounds at the $0.1 \mathrm{pb}$ level were found.

Estimating the reach of searches at the High Luminosity LHC (HL-LHC) with $3 \mathrm{ab}^{-1}$ of integrated luminosity is challenging because of the difficulty to reproduce multi-variate analyses and to envision improvements in future analysis techniques. Nevertheless, we can obtain some conservative estimates. A naive rescaling of the analysis in ref. [84] by the square root of the ratio of future to present integrated luminosities yields expected bounds at the $1-10 \mathrm{fb}$ level. Future improvements in the analysis will certainly lead to lower upper bounds.

With more data, other decay modes become competitive or even yield a stronger sensitivity. Examples include the channels $H \rightarrow t_{4} t \rightarrow Z \bar{t} t \rightarrow \ell^{+} \ell^{-} \bar{t} t(\ell=e, \mu)$ with hadronic tops and $H \rightarrow b_{4} b \rightarrow Z \bar{b} b \rightarrow \ell^{+} \ell^{-} \bar{b} b(\ell=e, \mu)$. In this case there is no missing energy (as opposite to the analysis in ref. [84]) and all invariant masses can be reconstructed. We implemented the new physics model in FeynRules [89]. We generated parton level events with MadGraph5 [90] and used Pythia8 [91, 92] and Delphes [93] for shower, hadronization and detector simulation. As a representative example we choose $m_{H}=2 \mathrm{TeV}$ and $m_{t_{4}, b_{4}}=1.5 \mathrm{TeV}$. In the $t_{4} t$ case, we require $H_{T}>1 \mathrm{TeV}$, two leptons with total transverse momentum $P_{T}(\ell \ell)>450 \mathrm{GeV}$, two fat-jets (as reconstructed by Delphes in its default setting) and the invariant mass of the di-lepton pair plus the hardest fat-jet $m_{j \ell \ell} \in[1.3,1.6] \mathrm{TeV}$. Similarly, in the $b_{4} b$ case, we require $H_{T}>1 \mathrm{TeV}$, two leptons 

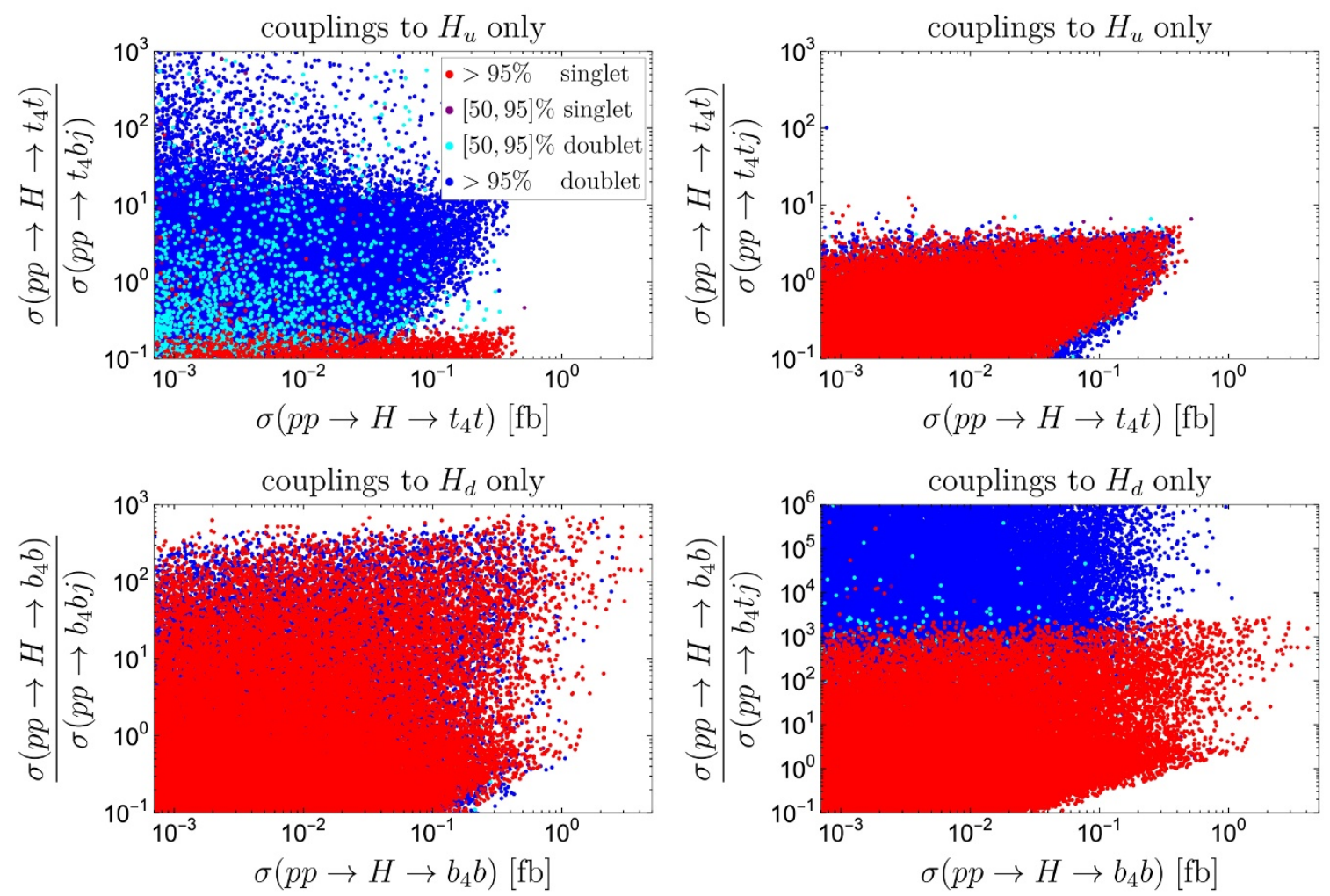

Figure 9. Ratios of production rates of $t_{4}$ (top) and $b_{4}$ (bottom) in heavy Higgs cascade decays and single production modes. Results for scenarios with couplings to both $H_{u}$ and $H_{d}$ are similar.

with total transverse momentum $P_{T}(\ell \ell)>550 \mathrm{GeV}$, two jets out of which at least one is $b$-tagged and $m_{j \ell \ell} \in[1.2,1.6] \mathrm{TeV}$, where $j$ is the hardest jet (whether $b$-tagged or not).

The resulting $m_{j \ell \ell}$ distributions are displayed in figure 8 where the signals are normalized to $1 \mathrm{fb}$ (prior to the $Z$ decay). From these results we estimate that the HL-LHC with an integrated luminosity of $3 \mathrm{ab}^{-1}$ is sensitive to cross sections: $\sigma\left(p p \rightarrow H \rightarrow t_{4} t \rightarrow\right.$ $Z t t) \gtrsim 0.19 \mathrm{fb}$ and $\sigma\left(p p \rightarrow H \rightarrow b_{4} b \rightarrow Z b b\right) \gtrsim 0.16 \mathrm{fb}$. We stress that this is a naive cutbased analysis which focuses on vectorlike quarks decaying to leptonic $Z$. We reasonably expect that, with a more sophisticated analysis and by considering/combining more decay channels, significantly smaller cross sections could be probed.

Finally, we would like to compare the production rates of vectorlike quarks in Higgs cascade decays and single production. Both mechanisms depend on the structure of Yukawa couplings and either process can dominate. This is illustrated in figure 9, where we plot the ratios of rates for these two production mechanisms. For the single production we consider both the $W$ mediated modes, $p p \rightarrow t_{4} b j$ and $p p \rightarrow b_{4} t j$, and the $Z$ mediated modes, $p p \rightarrow t_{4} t j$ and $p p \rightarrow b_{4} b j$. Note that $t_{4}\left(b_{4}\right)$ single production is typically dominated by the $W(Z)$ mode. We see that production rates of vectorlike quarks in Higgs cascade decays can easily be orders of magnitude larger than the single production rates. For the $b_{4}$, this result does not depend on its doublet/singlet nature, while for the $t_{4}$ it is more typical 
for the doublet case. ${ }^{4}$ These findings further motivate dedicated analyses for resonances decaying into $t_{4} t$ or $b_{4} b$.

In contrast to Higgs cascade decays and single production mechanisms, the pair production cross section of vectorlike quarks is model independent. Projections for the reach of the HL-LHC can be found in section 6.3.2 of ref. [6] where it is shown that vectorlike quarks with masses up to about $1350 \mathrm{GeV}$ can be discovered at the $5 \sigma$ level. The corresponding $2 \sigma$ sensitivity would extend to only somewhat larger masses because of the steep decrease of the pair production cross section for increasing vectorlike quark mass. We see that the experimental sensitivities of the HL-LHC to vectorlike quarks in heavy Higgs cascade decays and pair production are comparable.

The cascade decays of a heavy Higgs boson through vectorlike quarks provide an interesting opportunity to discover two new particles simultaneously. Not only these decay modes are sizable or can even dominate, but the usually dominant decay modes, $H \rightarrow t \bar{t}$ or $b \bar{b}$, are extremely challenging due to huge standard model backgrounds. In addition, for these decay modes, the resonant peak can be destroyed by the interference with the SM background [94].

\section{Conclusions}

We studied cascade decays of a heavy neutral Higgs boson through vectorlike quarks, $H \rightarrow t_{4} t \rightarrow W b t, Z t t, h t t$ and $H \rightarrow b_{4} b \rightarrow W t b, Z b b, h b b$, where $t_{4}\left(b_{4}\right)$ is the new up-type (down-type) quark mass eigenstate. Limiting the size of Yukawa couplings of vectorlike fields to one, in the two Higgs doublet model type-II, we found that these decay modes can be significant or can even dominate.

We found that the branching ratio of $H \rightarrow t_{4} t$ is larger than $10 \%$ for $\tan \beta \in[0.5,10]$ and can reach up to $40 \%$. More importantly, the branching ratio of $H \rightarrow b_{4} b$ is larger than $10 \%$ for any $\tan \beta>0.8$ and this mode can dominate for $\tan \beta \in[4,18]$ reaching up to $95 \%$. Multiplying with the Higgs production cross section, we found that $\sigma\left(p p \rightarrow H \rightarrow t_{4} t\right)$ is the largest at very small $\tan \beta$ and that, while the $H \rightarrow b_{4} b$ mode can dominate at medium $\tan \beta$, the $\sigma\left(p p \rightarrow H \rightarrow b_{4} b\right)$ can still be larger at both small and very large $\tan \beta$.

The lightest new quarks from heavy Higgs decays further decay into SM particles through $W, Z$ or $h$. We studied the correlations between the branching ratios of $H \rightarrow$ $t_{4} t\left(H \rightarrow b_{4} b\right)$ and individual branching ratios of $t_{4}\left(b_{4}\right)$. We presented the maximum production rates of individual final states in cascade decays of a heavy Higgs boson as functions of $m_{H}$ and $m_{t_{4}}$ or $m_{b_{4}}$. We found that, for Higgs cascade decays through a $t_{4}$, rates of $0.1 \mathrm{fb}$ extend up to $m_{H} \lesssim 2 \mathrm{TeV}$ and $m_{t_{4}} \lesssim 1.4 \mathrm{TeV}$. The rates above $0.01 \mathrm{fb}$ can be achieved for $m_{H} \lesssim 2.8 \mathrm{TeV}$ or $m_{t_{4}} \lesssim 2 \mathrm{TeV}$. For Higgs cascade decays through a $b_{4}$, rates of individual final states larger than $0.1 \mathrm{fb}$ extend up to $m_{H} \lesssim 2.5 \mathrm{TeV}$ and $m_{b_{4}} \lesssim 1.8 \mathrm{TeV}$

\footnotetext{
${ }^{4}$ In fact, for a pure doublet $t_{4}\left(b_{4}\right)$ in the scenario with couplings to $H_{u}\left(H_{d}\right)$ only, there is no $W t_{4} b$ $\left(W b_{4} t\right)$ vertex, implying the absence of the $W$ mediated single production. This is clearly visible in the upper-left and lower-right panels of figure 9. In scenarios with couplings to both $H_{u}$ and $H_{d}$ these $W$ couplings are not constrained to vanish and the blue points in these plots would extend to smaller values.
} 
and can be even larger than $1 \mathrm{fb}$ for $m_{H} \lesssim 1.6 \mathrm{TeV}$ and $m_{b_{4}} \lesssim 1.2 \mathrm{TeV}$. The rates above $0.01 \mathrm{fb}$ can be achieved for $m_{H} \lesssim 3.3 \mathrm{TeV}$ or $m_{b_{4}} \lesssim 2.4 \mathrm{TeV}$.

The signatures of cascade decays of a heavy Higgs boson through vectorlike quarks are almost identical to the production of any new resonance decaying to $t_{4} t$ or $b_{4} b$. They are also very similar to single productions of vectorlike quarks. However the topology of cascade decays provides more handles on the final states and thus dedicated searches have a potential to considerably improve the limits found in standard single production studies. So far, only searches for a resonance decaying to $t_{4} t$ have been performed. We have found that the rates for cascade decays through $b_{4}$ can be almost an order of magnitude larger than the rates for cascade decays through $t_{4}$ which motivates similar searches for a resonance decaying to $b_{4} b$.

We explored the reach of decay modes in which there is no missing energy and all invariant masses can be reconstructed. As examples we considered the channels $H \rightarrow t_{4} t \rightarrow$ $Z \bar{t} t \rightarrow \ell^{+} \ell^{-} \bar{t} t(\ell=e, \mu)$ with hadronic tops and $H \rightarrow b_{4} b \rightarrow Z \bar{b} b \rightarrow \ell^{+} \ell^{-} \bar{b} b(\ell=e, \mu)$. We have shown that even a very simple cut based analysis allows the HL-LHC with $3 \mathrm{ab}^{-1}$ of integrated luminosity to probe cross sections $\sigma\left(p p \rightarrow H \rightarrow t_{4} t \rightarrow Z t t\right) \gtrsim 0.19 \mathrm{fb}$ and $\sigma\left(p p \rightarrow H \rightarrow b_{4} b \rightarrow Z b b\right) \gtrsim 0.16 \mathrm{fb}$. We expect that, with more sophisticated analyses and by considering/combining more decay channels, significantly smaller cross sections could be tested.

We have also found that the rates for these processes can be much larger, even by orders of magnitude, than the rates for single productions of vectorlike quarks. The final states have significantly lower standard model backgrounds not only compared to single productions of vectorlike quarks but especially compared to the usually dominant decay modes of heavy Higgses, $H \rightarrow t \bar{t}$ or $b \bar{b}$, searches for which are extremely challenging. Thus, the cascade decays of a heavy Higgs boson through vectorlike quarks provide the best opportunities for the discovery of a new Higgs boson and vectorlike quarks.

\section{Acknowledgments}

SS thanks Bogdan Dobrescu and Eva Halkiadakis for insightful discussion. The work of RD was supported in part by the U.S. Department of Energy under grant number DE-SC0010120, and the IU Institute for Advanced Study. SS is supported by the National Research Foundation of Korea (NRF-2017R1D1A1B03032076 and in partial by NRF2018R1A4A1025334). This work was performed in part at the Aspen Center for Physics, which is supported by National Science Foundation grant PHY-1607611. SS appreciates the hospitality of Fermi National Accelerator Laboratory and expresses a special thanks to the Mainz Institute for Theoretical Physics (MITP) of the DFG Cluster of Excellence PRISMA $^{+}$(Project ID 39083149), for its hospitality and support. This work was supported by IBS under the project code, IBS-R018-D1 (SS). 


\section{A Partial decay widths of the heavy Higgs boson}

The decay widths for $H \rightarrow t_{i} t_{j} \equiv \bar{t}_{i} t_{j}+\bar{t}_{j} t_{i}$ with $i \neq j$ and $i, j=3,4,5$ are given by:

$$
\begin{aligned}
\Gamma\left(H \rightarrow t_{i} t_{j}\right)=\frac{3 m_{H}}{16 \pi} & \left\{\left(\left|\lambda_{t_{i} t_{j}}^{H}\right|^{2}+\left|\lambda_{t_{j} t_{i}}^{H *}\right|^{2}\right)\left(1-\frac{m_{t_{i}}^{2}+m_{t_{j}}^{2}}{m_{H}^{2}}\right)+4 \lambda_{t_{i} t_{j}}^{H} \lambda_{t_{j} t_{i}}^{H *} \frac{m_{t_{i}} m_{t_{j}}}{m_{H}^{2}}\right\} \\
& \times \sqrt{\lambda\left(1,\left(m_{t_{i}} / m_{H}\right)^{2},\left(m_{t_{j}} / m_{H}\right)^{2}\right)},
\end{aligned}
$$

where the couplings $\lambda_{t_{i} t_{j}}^{H}$ are given in eq. (A.48) of ref. [1] and $\lambda(x, y, z)=x^{2}+y^{2}+z^{2}-$ $2 x y-2 y z-2 z x$.

The decay widths for $H \rightarrow \bar{t}_{i} t_{i}$ with $i=3,4,5$ are given by:

$$
\Gamma\left(H \rightarrow t_{i} t_{i}\right)=\frac{3 m_{H}}{16 \pi}\left|\lambda_{t_{i} t_{i}}^{H}\right|^{2}\left(1-\frac{4 m_{t_{i}}^{2}}{m_{H}^{2}}\right)^{3 / 2} .
$$

Note that the corresponding partial widths into $b_{i}(i=3,4,5)$ can be obtained from eqs. (A.1) and (A.2) by replacing $t_{i, j} \rightarrow b_{i, j}$. For the $H \rightarrow \bar{t} t$ case we include the NLO correction factor $\left[1+\frac{4}{3} \frac{\alpha_{s}}{\pi} \Delta_{H}^{t}\left(\beta_{t}\right)\right]$ where $\beta_{t}=1-4 m_{t}^{2} / m_{H}^{2}$ and $\Delta_{H}^{t}\left(\beta_{t}\right)$ is given in eq. (2.14) of ref. [95].

The decay width for $H \rightarrow \bar{b} b$ in the $m_{b} \ll m_{H}$ limit is given by:

$$
\Gamma(H \rightarrow \bar{b} b)=\frac{3 m_{H}}{16 \pi}\left|\lambda_{b b}^{H}\right|^{2}\left[1+\Delta_{q q}+\Delta_{H}^{2}\right]
$$

where the coupling $\lambda_{b b}^{H}$ should be evaluated at a scale $O\left(m_{H}\right)$ and the $\mathrm{N}^{3} \mathrm{LO}$ correction factors, $\Delta_{q q}$ and $\Delta_{H}^{2}$, are presented in eqs. (2.11) and (2.12) of ref. [95].

The decay width of $H \rightarrow g g$ is given by:

$$
\begin{gathered}
\Gamma(H \rightarrow g g)=\frac{G_{F} \alpha_{s}^{2} m_{H}^{3}}{36 \sqrt{2} \pi^{3}} \cdot \frac{9}{16} \mid- \\
A_{1 / 2}^{t} \cot \beta+A_{1 / 2}^{b} \tan \beta+\frac{\lambda_{t_{4} t_{4}}^{H} v}{m_{t_{4}}} A_{1 / 2}^{t_{4}}+\frac{\lambda_{t_{5} t_{5}}^{H} v}{m_{t_{5}}} A_{1 / 2}^{t_{5}} \\
+\frac{\lambda_{b_{4} b_{4}}^{H} v}{m_{b_{4}}} A_{1 / 2}^{b_{4}}+\left.\frac{\lambda_{b_{5} b_{5}}^{H} v}{m_{b_{5}}} A_{1 / 2}^{b_{5}}\right|^{2}
\end{gathered}
$$

where $A_{1 / 2}^{q}=2\left[\tau_{q}+\left(\tau_{q}-1\right) f\left(\tau_{q}\right)\right] / \tau_{q}^{2}$ with $\tau_{q}=m_{H}^{2} / 4 m_{q}^{2}$ and

$$
f(\tau)=\left\{\begin{array}{ll}
\arcsin ^{2} \sqrt{\tau}, & \tau \leq 1 \\
-\frac{1}{4}\left[\log \frac{1+\sqrt{1-\tau^{-1}}}{1-\sqrt{1-\tau^{-1}}}-i \pi\right]^{2}, & \tau>1
\end{array} .\right.
$$

Note that the contributions of vectorlike quarks have been included following the prescription discussed in refs. [96, 97]. 
The decay width for $H \rightarrow \gamma \gamma$ can be obtained from eq. (2.23) of ref. [68] supplemented by the presecription discussed in refs. [96, 97] and is given by:

$$
\begin{aligned}
\Gamma(H \rightarrow \gamma \gamma)=\frac{9 G_{F} \alpha^{2} m_{H}^{3}}{128 \sqrt{2} \pi^{3}} & \mid\left(\frac{2}{3}\right)^{2}(-\cot \beta) \cdot A_{1 / 2}\left(\tau_{t}\right)+\left(\frac{1}{3}\right)^{2} \tan \beta \cdot A_{1 / 2}\left(\tau_{b}\right) \\
+ & \left(\frac{2}{3}\right)^{2} \cdot \frac{\lambda_{t_{4} t_{4}}^{H} v}{m_{t_{4}}} \cdot A_{1 / 2}\left(\tau_{t_{4}}\right)+\left(\frac{2}{3}\right)^{2} \cdot \frac{\lambda_{t_{5} t_{5}}^{H} v}{m_{t_{5}}} \cdot A_{1 / 2}\left(\tau_{t_{5}}\right) \\
+ & \left(\frac{1}{3}\right)^{2} \cdot \frac{\lambda_{b_{4} b_{4}}^{H} v}{m_{b_{4}}} \cdot A_{1 / 2}\left(\tau_{b_{4}}\right)+\left.\left(\frac{1}{3}\right)^{2} \cdot \frac{\lambda_{b_{5} b_{5}}^{H} v}{m_{b_{5}}} \cdot A_{1 / 2}\left(\tau_{b_{5}}\right)\right|^{2} .
\end{aligned}
$$

Finally, the decay width for $H \rightarrow h h$, where $h$ is the SM-like Higgs boson, is given by:

$$
\Gamma(H \rightarrow h h)=\frac{9 G_{F}}{16 \pi \sqrt{2}} \frac{M_{Z}^{4}}{m_{H}} \sin ^{2}(2 \beta) \cos ^{2}(2 \beta) \sqrt{1-4 \frac{m_{h}^{2}}{m_{H}^{2}}} .
$$

Open Access. This article is distributed under the terms of the Creative Commons Attribution License (CC-BY 4.0), which permits any use, distribution and reproduction in any medium, provided the original author(s) and source are credited.

\section{References}

[1] R. Dermíšek, E. Lunghi and S. Shin, Hunting for Vectorlike Quarks, JHEP 04 (2019) 019 [arXiv: 1901.03709] [INSPIRE].

[2] R. Dermisek, E. Lunghi and S. Shin, Two Higgs doublet model with vectorlike leptons and contributions to $p p \rightarrow W W$ and $H \rightarrow W W$, JHEP 02 (2016) 119 [arXiv:1509.04292] [INSPIRE].

[3] R. Dermisek, E. Lunghi and S. Shin, Contributions of flavor violating couplings of a Higgs boson to $p p \rightarrow W W$, JHEP 08 (2015) 126 [arXiv:1503.08829] [INSPIRE].

[4] R. Dermisek, E. Lunghi and S. Shin, New decay modes of heavy Higgs bosons in a two Higgs doublet model with vectorlike leptons, JHEP 05 (2016) 148 [arXiv: 1512.07837] [INSPIRE].

[5] R. Dermisek, E. Lunghi and S. Shin, New constraints and discovery potential for Higgs to Higgs cascade decays through vectorlike leptons, JHEP 10 (2016) 081 [arXiv:1608.00662] [INSPIRE].

[6] X. Cid Vidal et al., Report from Working Group 3, CERN Yellow Rep. Monogr. 7 (2019) 585 [arXiv: 1812.07831] [INSPIRE].

[7] R. Dermisek, J.P. Hall, E. Lunghi and S. Shin, Limits on Vectorlike Leptons from Searches for Anomalous Production of Multi-Lepton Events, JHEP 12 (2014) 013 [arXiv:1408.3123] [INSPIRE].

[8] D. Choudhury, T.M.P. Tait and C.E.M. Wagner, Beautiful mirrors and precision electroweak data, Phys. Rev. D 65 (2002) 053002 [hep-ph/0109097] [InSPIRE].

[9] R. Dermisek, S.-G. Kim and A. Raval, New Vector Boson Near the Z-pole and the Puzzle in Precision Electroweak Data, Phys. Rev. D 84 (2011) 035006 [arXiv:1105. 0773] [INSPIRE].

[10] R. Dermisek, S.-G. Kim and A. Raval, Z' near the Z-pole, Phys. Rev. D 85 (2012) 075022 [arXiv:1201.0315] [INSPIRE]. 
[11] B. Batell, S. Gori and L.-T. Wang, Higgs Couplings and Precision Electroweak Data, JHEP 01 (2013) 139 [arXiv: 1209.6382] [InSPIRE].

[12] K. Kannike, M. Raidal, D.M. Straub and A. Strumia, Anthropic solution to the magnetic muon anomaly: the charged see-saw, JHEP 02 (2012) 106 [Erratum ibid. 10 (2012) 136] [arXiv:1111.2551] [INSPIRE].

[13] R. Dermisek and A. Raval, Explanation of the Muon g-2 Anomaly with Vectorlike Leptons and its Implications for Higgs Decays, Phys. Rev. D 88 (2013) 013017 [arXiv:1305.3522] [INSPIRE].

[14] K.S. Babu and J.C. Pati, The Problems of unification mismatch and low $\alpha^{3}$ : A Solution with light vector-like matter, Phys. Lett. B 384 (1996) 140 [hep-ph/9606215] [INSPIRE].

[15] C.F. Kolda and J. March-Russell, Low-energy signatures of semiperturbative unification, Phys. Rev. D 55 (1997) 4252 [hep-ph/9609480] [INSPIRE].

[16] D. Ghilencea, M. Lanzagorta and G.G. Ross, Strong unification, Phys. Lett. B 415 (1997) 253 [hep-ph/9707462] [INSPIRE].

[17] G. Amelino-Camelia, D. Ghilencea and G.G. Ross, The Effect of Yukawa couplings on unification predictions and the nonperturbative limit, Nucl. Phys. B 528 (1998) 35 [hep-ph/9804437] [INSPIRE].

[18] M. Bastero-Gil and B. Brahmachari, Semiperturbative unification with extra vector-like families, Nucl. Phys. B 575 (2000) 35 [hep-ph/9907318] [InSPIRE].

[19] R. Dermisek, Insensitive Unification of Gauge Couplings, Phys. Lett. B 713 (2012) 469 [arXiv: 1204.6533] [INSPIRE].

[20] R. Dermisek, Unification of gauge couplings in the standard model with extra vectorlike families, Phys. Rev. D 87 (2013) 055008 [arXiv:1212.3035] [InSPIRE].

[21] R. Dermisek and N. McGinnis, Mass scale of vectorlike matter and superpartners from IR fixed point predictions of gauge and top Yukawa couplings, Phys. Rev. D 97 (2018) 055009 [arXiv: 1712.03527] [INSPIRE].

[22] R. Dermíšek and N. McGinnis, Top-bottom-tau Yukawa coupling unification in the MSSM plus one vectorlike family and fermion masses as IR fixed points, Phys. Rev. D 99 (2019) 035033 [arXiv: 1810.12474] [INSPIRE].

[23] R. Dermisek, Loop suppressed electroweak symmetry breaking and naturally heavy superpartners, Phys. Rev. D 95 (2017) 015002 [arXiv:1606.09031] [INSPIRE].

[24] T. Moroi and Y. Okada, Radiative corrections to Higgs masses in the supersymmetric model with an extra family and antifamily, Mod. Phys. Lett. A 7 (1992) 187 [InSPIRE].

[25] T. Moroi and Y. Okada, Upper bound of the lightest neutral Higgs mass in extended supersymmetric Standard Models, Phys. Lett. B 295 (1992) 73 [INSPIRE].

[26] K.S. Babu, I. Gogoladze, M.U. Rehman and Q. Shafi, Higgs Boson Mass, Sparticle Spectrum and Little Hierarchy Problem in Extended MSSM, Phys. Rev. D 78 (2008) 055017 [arXiv: 0807.3055] [INSPIRE].

[27] S.P. Martin, Extra vector-like matter and the lightest Higgs scalar boson mass in low-energy supersymmetry, Phys. Rev. D 81 (2010) 035004 [arXiv:0910.2732] [INSPIRE].

[28] P.W. Graham, A. Ismail, S. Rajendran and P. Saraswat, A Little Solution to the Little Hierarchy Problem: A Vector-like Generation, Phys. Rev. D 81 (2010) 055016 [arXiv:0910.3020] [INSPIRE]. 
[29] S.P. Martin, Raising the Higgs Mass with Yukawa Couplings for Isotriplets in Vector-Like Extensions of Minimal Supersymmetry, Phys. Rev. D 82 (2010) 055019 [arXiv:1006.4186] [INSPIRE].

[30] A. Falkowski and R. Vega-Morales, Exotic Higgs decays in the golden channel, JHEP 12 (2014) 037 [arXiv: 1405.1095] [INSPIRE].

[31] R. Dermisek, A. Raval and S. Shin, Effects of vectorlike leptons on $h \rightarrow 4 \ell$ and the connection to the muon g-2 anomaly, Phys. Rev. D 90 (2014) 034023 [arXiv:1406.7018] [INSPIRE].

[32] R. Dermíšek and N. McGinnis, Seven largest couplings of the standard model as IR fixed points, Phys. Rev. Lett. 122 (2019) 181803 [arXiv:1812.05240] [INSPIRE].

[33] J.A. Aguilar-Saavedra, R. Benbrik, S. Heinemeyer and M. Pérez-Victoria, Handbook of vectorlike quarks: Mixing and single production, Phys. Rev. D 88 (2013) 094010 [arXiv: 1306.0572] [INSPIRE].

[34] B.A. Dobrescu and C.T. Hill, Electroweak symmetry breaking via top condensation seesaw, Phys. Rev. Lett. 81 (1998) 2634 [hep-ph/9712319] [INSPIRE].

[35] R.S. Chivukula, B.A. Dobrescu, H. Georgi and C.T. Hill, Top Quark Seesaw Theory of Electroweak Symmetry Breaking, Phys. Rev. D 59 (1999) 075003 [hep-ph/9809470] [INSPIRE].

[36] H.-J. He, C.T. Hill and T.M.P. Tait, Top Quark Seesaw, Vacuum Structure and Electroweak Precision Constraints, Phys. Rev. D 65 (2002) 055006 [hep-ph/0108041] [INSPIRE].

[37] C.T. Hill and E.H. Simmons, Strong Dynamics and Electroweak Symmetry Breaking, Phys. Rept. 381 (2003) 235 [Erratum ibid. 390 (2004) 553] [hep-ph/0203079] [INSPIRE].

[38] K. Agashe, R. Contino and A. Pomarol, The Minimal composite Higgs model, Nucl. Phys. B 719 (2005) 165 [hep-ph/0412089] [INSPIRE].

[39] R. Contino, L. Da Rold and A. Pomarol, Light custodians in natural composite Higgs models, Phys. Rev. D 75 (2007) 055014 [hep-ph/0612048] [INSPIRE].

[40] R. Barbieri, B. Bellazzini, V.S. Rychkov and A. Varagnolo, The Higgs boson from an extended symmetry, Phys. Rev. D 76 (2007) 115008 [arXiv:0706.0432] [INSPIRE].

[41] C. Anastasiou, E. Furlan and J. Santiago, Realistic Composite Higgs Models, Phys. Rev. D 79 (2009) 075003 [arXiv:0901.2117] [InSPIRE].

[42] G. Cacciapaglia, H. Cai, A. Deandrea and A. Kushwaha, Composite Higgs and Dark Matter Model in SU(6)/SO(6), JHEP 10 (2019) 035 [arXiv: 1904.09301] [INSPIRE].

[43] C. Cai, G. Cacciapaglia and H.-H. Zhang, Vacuum alignment in a composite 2HDM, JHEP 01 (2019) 130 [arXiv: 1805.07619] [INSPIRE].

[44] T. Ma and G. Cacciapaglia, Fundamental Composite 2HDM: SU(N) with 4 flavours, JHEP 03 (2016) 211 [arXiv: 1508.07014] [INSPIRE].

[45] J. Serra, Beyond the Minimal Top Partner Decay, JHEP 09 (2015) 176 [arXiv:1506.05110] [INSPIRE].

[46] E. Bertuzzo, T.S. Ray, H. de Sandes and C.A. Savoy, On Composite Two Higgs Doublet Models, JHEP 05 (2013) 153 [arXiv:1206.2623] [INSPIRE].

[47] J. Mrazek, A. Pomarol, R. Rattazzi, M. Redi, J. Serra and A. Wulzer, The Other Natural Two Higgs Doublet Model, Nucl. Phys. B 853 (2011) 1 [arXiv:1105.5403] [InSPIRE].

[48] N. Arkani-Hamed, A.G. Cohen, E. Katz and A.E. Nelson, The Littlest Higgs, JHEP 07 (2002) 034 [hep-ph/0206021] [INSPIRE]. 
[49] M. Schmaltz and D. Tucker-Smith, Little Higgs review, Ann. Rev. Nucl. Part. Sci. 55 (2005) 229 [hep-ph/0502182] [INSPIRE].

[50] A. Davidson and K.C. Wali, Family Mass Hierarchy From Universal Seesaw Mechanism, Phys. Rev. Lett. 60 (1988) 1813 [InSPIRE].

[51] K.S. Babu and R.N. Mohapatra, A Solution to the Strong CP Problem Without an Axion, Phys. Rev. D 41 (1990) 1286 [inSPIRE].

[52] B. Grinstein, M. Redi and G. Villadoro, Low Scale Flavor Gauge Symmetries, JHEP 11 (2010) 067 [arXiv: 1009.2049] [InSPIRE].

[53] D. Guadagnoli, R.N. Mohapatra and I. Sung, Gauged Flavor Group with Left-Right Symmetry, JHEP 04 (2011) 093 [arXiv: 1103.4170] [INSPIRE].

[54] J.A. Aguilar-Saavedra, Identifying top partners at LHC, JHEP 11 (2009) 030 [arXiv: 0907.3155] [INSPIRE].

[55] Y. Okada and L. Panizzi, LHC signatures of vector-like quarks, Adv. High Energy Phys. 2013 (2013) 364936 [arXiv:1207.5607] [INSPIRE].

[56] A. De Simone, O. Matsedonskyi, R. Rattazzi and A. Wulzer, A First Top Partner Hunter's Guide, JHEP 04 (2013) 004 [arXiv:1211.5663] [INSPIRE].

[57] M. Buchkremer, G. Cacciapaglia, A. Deandrea and L. Panizzi, Model Independent Framework for Searches of Top Partners, Nucl. Phys. B 876 (2013) 376 [arXiv:1305.4172] [InSPIRE].

[58] H.E. Haber, Nonminimal Higgs sectors: The Decoupling limit and its phenomenological implications, in Joint U.S.-Polish Workshop on Physics from Planck Scale to Electro-Weak Scale (SUSY 94), Warsaw, Poland, 21-24 September 1994, pp. 1-16 (1994) [hep-ph/9501320] [INSPIRE].

[59] J.F. Gunion and H.E. Haber, The CP conserving two Higgs doublet model: The Approach to the decoupling limit, Phys. Rev. D 67 (2003) 075019 [hep-ph/0207010] [INSPIRE].

[60] J. Bernon, J.F. Gunion, H.E. Haber, Y. Jiang and S. Kraml, Scrutinizing the alignment limit in two-Higgs-doublet models: $m_{h}=125 \mathrm{GeV}$, Phys. Rev. D 92 (2015) 075004 [arXiv: 1507.00933] [INSPIRE].

[61] Particle Data Group collaboration, Review of Particle Physics, Phys. Rev. D 98 (2018) 030001 [INSPIRE].

[62] L. Lavoura and J.P. Silva, The Oblique corrections from vector-like singlet and doublet quarks, Phys. Rev. D 47 (1993) 2046 [INSPIRE].

[63] C.-Y. Chen, S. Dawson and E. Furlan, Vectorlike fermions and Higgs effective field theory revisited, Phys. Rev. D 96 (2017) 015006 [arXiv:1703.06134] [INSPIRE].

[64] C.P. Burgess, S. Godfrey, H. Konig, D. London and I. Maksymyk, Model independent global constraints on new physics, Phys. Rev. D 49 (1994) 6115 [hep-ph/9312291] [inSPIRE].

[65] CMS collaboration, Search for high-mass diphoton resonances in proton-proton collisions at $13 \mathrm{TeV}$ and combination with $8 \mathrm{TeV}$ search, Phys. Lett. B 767 (2017) 147 [arXiv: 1609.02507] [INSPIRE].

[66] ATLAS collaboration, Measurements of Higgs boson properties in the diphoton decay channel with $36 \mathrm{fb}^{-1}$ of pp collision data at $\sqrt{s}=13 \mathrm{TeV}$ with the ATLAS detector, Phys. Rev. D 98 (2018) 052005 [arXiv: 1802.04146] [inSPIRE].

[67] ATLAS collaboration, Combined measurements of Higgs boson production and decay using up to $80 \mathrm{fb}^{-1}$ of proton-proton collision data at $\sqrt{\mathrm{s}}=13 \mathrm{TeV}$ collected with the ATLAS experiment, ATLAS-CONF-2018-031 (2018). 
[68] A. Djouadi, The Anatomy of electro-weak symmetry breaking. II. The Higgs bosons in the minimal supersymmetric model, Phys. Rept. 459 (2008) 1 [hep-ph/0503173] [INSPIRE].

[69] N. Bizot and M. Frigerio, Fermionic extensions of the Standard Model in light of the Higgs couplings, JHEP 01 (2016) 036 [arXiv:1508.01645] [INSPIRE].

[70] ATLAS collaboration, Combination of the searches for pair-produced vector-like partners of the third-generation quarks at $\sqrt{s}=13$ TeV with the ATLAS detector, Phys. Rev. Lett. 121 (2018) 211801 [arXiv: 1808.02343] [INSPIRE].

[71] ATLAS collaboration, Search for pair production of heavy vector-like quarks decaying to high- $p_{\mathrm{T}} W$ bosons and $b$ quarks in the lepton-plus-jets final state in pp collisions at $\sqrt{s}=13 \mathrm{TeV}$ with the ATLAS detector, hepdata.77271 (2017).

[72] CMS collaboration, Search for single production of vector-like quarks decaying into a b quark and a $W$ boson in proton-proton collisions at $\sqrt{s}=13$ TeV, Phys. Lett. B 772 (2017) 634 [arXiv: 1701.08328] [INSPIRE].

[73] ATLAS collaboration, Search for single production of a vector-like B quark decaying into a bottom quark and a Higgs boson which decays into a pair of photons, ATLAS-CONF-2018-024 (2018).

[74] ATLAS collaboration, Search for additional heavy neutral Higgs and gauge bosons in the ditau final state produced in $36 \mathrm{fb}^{-1}$ of pp collisions at $\sqrt{\mathrm{s}}=13 \mathrm{TeV}$ with the ATLAS detector, JHEP 01 (2018) 055 [arXiv: 1709.07242] [INSPIRE].

[75] ATLAS collaboration, Search for additional heavy neutral Higgs and gauge bosons in the ditau final state produced in $36 \mathrm{fb}^{-1}$ of pp collisions at $\sqrt{\mathrm{s}}=13 \mathrm{TeV}$ with the ATLAS detector, hepdata.78402 (2018).

[76] ATLAS collaboration, Search for new phenomena in high-mass diphoton final states using $37 \mathrm{fb}^{-1}$ of proton-proton collisions collected at $\sqrt{s}=13 \mathrm{TeV}$ with the ATLAS detector, Phys. Lett. B 775 (2017) 105 [arXiv: 1707.04147] [INSPIRE].

[77] ATLAS collaboration, Search for new phenomena in high-mass diphoton final states using $37 \mathrm{fb}^{-1}$ of proton-proton collisions collected at $\sqrt{s}=13 \mathrm{TeV}$ with the ATLAS detector, hepdata.79924 (2017).

[78] ATLAS collaboration, Search for pair production of Higgs bosons in the $b \bar{b} b \bar{b}$ final state using proton-proton collisions at $\sqrt{s}=13 \mathrm{TeV}$ with the ATLAS detector, JHEP 01 (2019) 030 [arXiv: 1804.06174] [INSPIRE].

[79] R.V. Harlander, S. Liebler and H. Mantler, SusHi: A program for the calculation of Higgs production in gluon fusion and bottom-quark annihilation in the Standard Model and the MSSM, Comput. Phys. Commun. 184 (2013) 1605 [arXiv:1212.3249] [INSPIRE].

[80] D. Greco and D. Liu, Hunting composite vector resonances at the LHC: naturalness facing data, JHEP 12 (2014) 126 [arXiv:1410.2883] [INSPIRE].

[81] M. Backović, T. Flacke, B. Jain and S.J. Lee, LHC vector resonance searches in the $t \bar{t} Z$ final state, JHEP 03 (2017) 127 [arXiv: 1610.08810] [INSPIRE].

[82] C. Bini, R. Contino and N. Vignaroli, Heavy-light decay topologies as a new strategy to discover a heavy gluon, JHEP 01 (2012) 157 [arXiv:1110.6058] [INSPIRE].

[83] CMS collaboration, Search for single production of a vector-like T quark decaying to a $Z$ boson and a top quark in proton-proton collisions at $\sqrt{s}=13 \mathrm{TeV}$, Phys. Lett. B 781 (2018) 574 [arXiv: 1708.01062$]$ [INSPIRE]. 
[84] CMS collaboration, Search for a heavy resonance decaying to a top quark and a vector-like top quark in the lepton+jets final state in pp collisions at $\sqrt{s}=13 \mathrm{TeV}$, Eur. Phys. J. C 79 (2019) 208 [arXiv:1812.06489] [INSPIRE].

[85] CMS collaboration, Search for single production of vector-like quarks decaying to a $b$ quark and a Higgs boson, JHEP 06 (2018) 031 [arXiv:1802.01486] [INSPIRE].

[86] CMS collaboration, Search for single production of vector-like quarks decaying to a top quark and a $W$ boson in proton-proton collisions at $\sqrt{s}=13$ TeV, Eur. Phys. J. C 79 (2019) 90 [arXiv: 1809.08597] [INSPIRE].

[87] ATLAS collaboration, Search for pair- and single-production of vector-like quarks in final states with at least one $Z$ boson decaying into a pair of electrons or muons in pp collision data collected with the ATLAS detector at $\sqrt{s}=13$ TeV, Phys. Rev. D 98 (2018) 112010 [arXiv: 1806.10555] [INSPIRE].

[88] ATLAS collaboration, Search for single production of vector-like quarks decaying into Wb in pp collisions at $\sqrt{s}=13$ TeV with the ATLAS detector, JHEP 05 (2019) 164 [arXiv: 1812.07343] [INSPIRE].

[89] C. Degrande, C. Duhr, B. Fuks, D. Grellscheid, O. Mattelaer and T. Reiter, UFO - The Universal FeynRules Output, Comput. Phys. Commun. 183 (2012) 1201 [arXiv:1108.2040] [INSPIRE].

[90] J. Alwall et al., The automated computation of tree-level and next-to-leading order differential cross sections and their matching to parton shower simulations, JHEP 07 (2014) 079 [arXiv: 1405.0301] [INSPIRE].

[91] T. Sjöstrand, S. Mrenna and P.Z. Skands, PYTHIA 6.4 Physics and Manual, JHEP 05 (2006) 026 [hep-ph/0603175] [INSPIRE].

[92] T. Sjöstrand et al., An Introduction to PYTHIA 8.2, Comput. Phys. Commun. 191 (2015) 159 [arXiv:1410.3012] [INSPIRE].

[93] DELPHES 3 collaboration, DELPHES 3, A modular framework for fast simulation of a generic collider experiment, JHEP 02 (2014) 057 [arXiv: 1307.6346] [INSPIRE].

[94] S. Jung, J. Song and Y.W. Yoon, Dip or nothingness of a Higgs resonance from the interference with a complex phase, Phys. Rev. D 92 (2015) 055009 [arXiv:1505.00291] [INSPIRE].

[95] A. Djouadi, The Anatomy of electro-weak symmetry breaking. I: The Higgs boson in the standard model, Phys. Rept. 457 (2008) 1 [hep-ph/0503172] [INSPIRE].

[96] G. Cacciapaglia, A. Deandrea and J. Llodra-Perez, $H \rightarrow \gamma \gamma$ beyond the Standard Model, JHEP 06 (2009) 054 [arXiv: 0901.0927] [INSPIRE].

[97] G. Cacciapaglia, A. Deandrea, L. Panizzi, N. Gaur, D. Harada and Y. Okada, Heavy Vector-like Top Partners at the LHC and flavour constraints, JHEP 03 (2012) 070 [arXiv:1108.6329] [INSPIRE]. 